\title{
A Framework for Optimization of Technical Indicators Parameters for Forex (Foreign Exchange) based on Genetic Algorithm
}

\author{
Tamer Sh. Mazen \\ Dept. of Management Information Systems, \\ Modern Academy for Computer Science and \\ Information Technology, Cairo, Egypt
}

\begin{abstract}
A new methodology to optimize the parameters of a collection technical analysis indicators of Forex (Foreign Exchange) based genetic algorithm (GA), over two objective functions Sharpe ratio and annual profit is presented in this study. The technique handles four indicators DEMAC (Double Exponential Moving Average Crossovers), RSI (Relative Strength Index), MACD (Moving Average Convergence Divergence), and MARSI (Moving Average RSI) indicators. A proposed framework is tested on 20 years of historical data EURUSD. Results showed that the optimized parameters obtained by the proposed technique improved the profits obtained by the indicators with their typical parameters, the Buy and Hold strategy and the random strategy.
\end{abstract}

\section{Keywords}

Forex, Forex Indicators, Technical Analysis, Genetic Algorithms, Parameter Optimization.

\section{INTRODUCTION}

The process of forecasting currency prices is one of the most difficult operations. All currency dealers ex. (dealers, companies, trading, etc.) need to forecast currency rates. In the field of trading, traders seek the desire to determine the most appropriate time and price to enter or exit the market to achieve the highest possible profitability. The use of time series methodology had a great role in predicting prices, although its results are volatile and may lead to many losses.

Scientists in economics have created 3 different schools for forecasting financial markets. The first school is the Effective Market Hypothesis (EMH). It depends on the fact that stock market prices develop according to a random path. As it is not possible to predict future price movement based on past price movements [1] [2].

The second method is financial analysis. It is based on analyzing the financial statements of companies. This method requires a lot of accounting data and information.

This data is difficult to obtain permanently. And it often had negative results in small-sized companies. This analysis should not be used in the analysis of money markets and currency exchange, but it is used in the analysis of Shares of companies.Technical analysis is based on previous prices, as the analysis uses a set of technical indicators for analysis, which was formed according to a set of criteria. Technical indicators are a set of calculations based onsome mathematical and statistical theories, and traders use them to predict prices degrees of difficulty may vary between one indicator and the other. Most of the time, traders use indicators for two reasons (the first is to confirm the price movement and the second is to find signals to enter or exit the market). [3] Examples of technical indicators are RSI (Relative Strength Index), ROC
(Rate Of Change), Moving Averages, OBV (On Balance Volume) indicators, Moving Average Convergence Divergence (MACD) and Moving Average Relative Strength Index (MARSI) [CCI (Commodity Channel Index) [4] [5].

For attempts to improve the indicators' results, it was necessary to go in two trends, the first Generating new trading rules based on a collection of indicators. Second trend is optimizing the parameters of indicators.

Hirabayashi et al. [6] used Genetic Algorithms to generate new trading rules for the Foreign Exchange market.

SeungKyu Lee \& Byung-Ro Moon [7] developed a modular Genetic Programming to find attractive trading rules. They defined the attractive rule to be the one that is profitable, simple, and frequent.

Fernandez Blanco et al. proposed an Evolutionary Algorithm "EA" based technique to optimize the parameters of the Moving Average Convergence Divergence "MACD" indicator, also Both Adriano Simoes et al. and V.Kapoor et al. used Genetic Algorithms to optimize the parameters of Simple Moving Average Crossovers "SMVC" indicator [8][9] [10].

In this research GA is used to tuning indicator parameters over two objective annual profit and Sharpe ratio. while GA is used to find the best collection of parameters for each indicator.

The best parameters that provide the best buy/sell signals, that provide the highest profits.

This research handled four different indicators Moving Average Convergence/Divergence (MACD), Double Exponential Moving Average crossovers (DEMAC), Relative Strength Index (RSI) indicator and finally, the Moving Average RSI indicator (MARSI).

The performance of the optimized indicators is calculated against the Buy and Hold strategy. The Buy and Hold is a passive investment strategy, which stocks are bought and held for a long period of time. That regardless of the fluctuations in the market. the random strategy, and the indicators with their typical parameters. [11].

\section{BACKGROUND}

This section describes genetic algorithm "GA" and the indicators that are used.

\subsection{Genetic Algorithms}

Genetic Algorithm (GA) is a search-based optimization technique on the principles of Genetics and Natural Selection. It is frequently used to find nearest optimal solutions for problems. that problems would take a lifetime to solve. 
Fig 1. shows the basic steps of Genetic Algorithms (GAs). First, a population of random solutions is provided. For each chromosome in the population fitness function is evaluated. The chromosomes with the highest fitness values are more probably to be selected for reproduction using crossover and mutation. If the stopping criteria are not met the procedure is repeated by evaluating again the new population and so on.

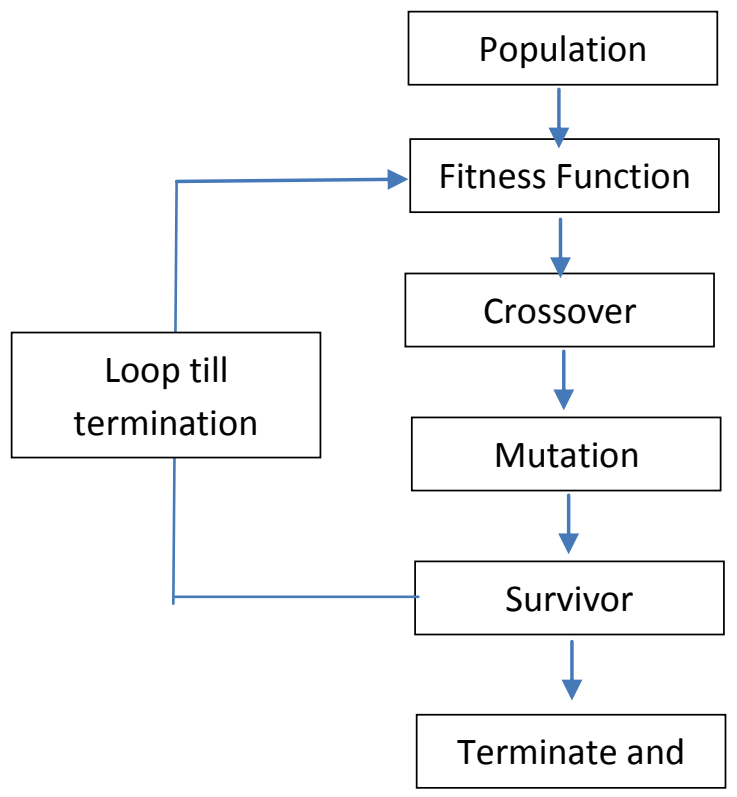

Fig. 1 Genetic Algorithm Steps

\subsection{Forex indicators}

These indicators are RSI (Relative Strength Index), DEMAC (Double Exponential Moving Average Crossovers), MARSI (Moving Average Relative Strength Index) and MACD (Moving Average Convergence Divergence).

\subsubsection{Double Exponential Moving Average Crossovers DEMAC}

A moving average is used to get an overview of market direction. It is preferred to be used in predicting long-term trends. It is most often used by financial market analysts on long-term periods of 50 or 200 days, while in short-term periods it is calculated by 7, 14 and 30 days. [12] [13] [14]

A moving average represents the average value of data over a specific time period. There are several types of moving averages, among which are the most common of which are the simple moving average (SMA) and the Exponential moving average (EMA). [12] [13] [14]

SMA is calculated by computing an average of close of $\mathrm{N}$ days, ass seen in (1), that average is moving because at the end of each trading day, the last day is added, while the earliest day of the previous average is dropped. [12] [13] [14] [15]

$S M A_{(i)}=\frac{\sum_{i=1}^{n} \operatorname{close}(i)}{n}$

Where $n$ is the period for calculating the average, close is closing price for day $i$

Equation (2) represent a calculation of EMA, that placed a greater weight and significance on the most recent data points.

$E M A_{i}=\left(\right.$ close $_{i} \times\left(\right.$ weight $\left._{i}\right)+$ weight $_{M A} \times E M A(i-1)$ weight $_{i}=\frac{2}{n+1}$

(2)

weight $_{i}=1-$ weight $_{i}$

Where $i$ is current day, $n$ is the period for calculating the average, weight $_{i}$ is the weight assigned to the last day of calculation (day) and weight ${ }_{M A}$ is the weight assigned to the previous EMA.

This study is based on trading using double EMA crossover. when short-term moving average is crossed up the long-term moving average, a buy signal is generated. While the opposite is happened a sell, signal is generated. As seen in Fig. 2blue curve line is used for $E M A_{\text {short-term }}$ and red curve line for $E M A_{\text {short-term }}$.
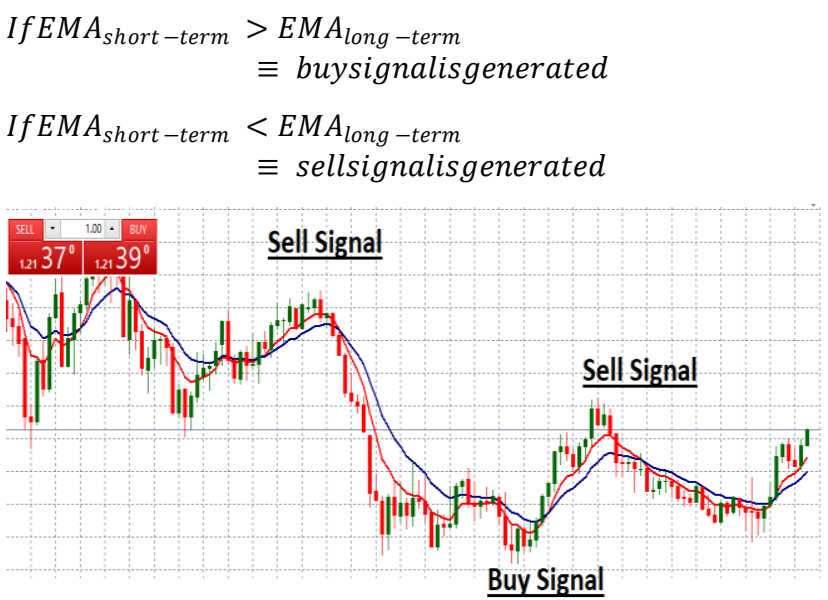

Fig. 2 Buy \& Sell Signals [12]

\subsubsection{Relative Strength Index. (RSI)}

Most of traders and technical analysist are depended on RSI where it can be useful tool depending on the type of trading. It is used to check how well a currency has performed over a certain period of time. It is based on measuring the fluctuation between the volume of price movements and the speed of movement in the price [16]. Equation (3) used to calculate RSI. $R s=\frac{\text { the total gains calculated over the last } \mathrm{n} \text { days }}{\mathrm{n} \text { days are the total losses calculated over the last } \mathrm{n} \text { days }}$

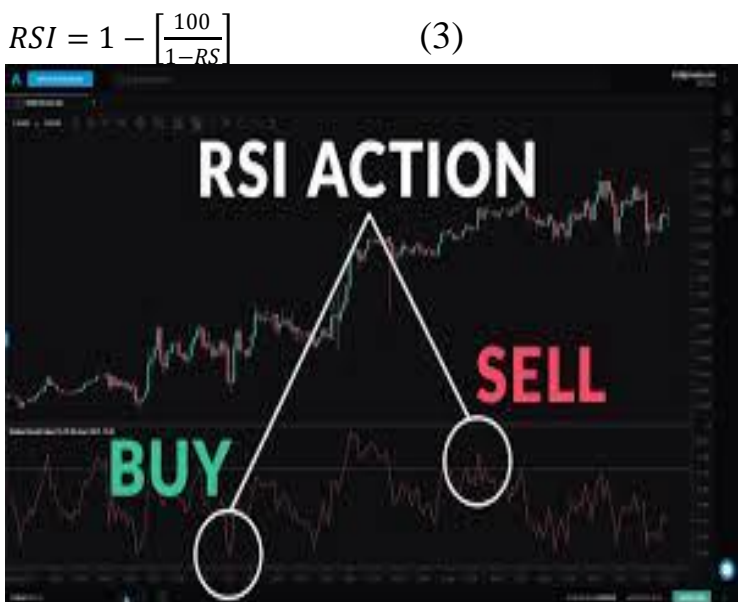

Fig. 3RSI Signals [16]

Fig. 3, represent RSI indictor signals. An Expertise of stock market analysis detriment RSI levels, 30,50 and 70. 
Expertise level based on calculation for 14 days period.

If RSI comes over 30 and cross over 30, be ready to buy or buy part of your plan.

If RSI comes below 30 and cross over 30, buy all of your plan.

If RSI comes below 70 and cross over 70 , be ready to sell or gain part of profit.

If RSI comes over 70 and cross over 70, sell all quantity.

\subsubsection{Moving average RSI (MRSI)}

It is a combination between RSI and Simple Moving Average (SMV). it used to make RSI indicator smooth out. In RSI, buying or selling when RSI crosses the thresholds while in MRSI, Buy and sell signals are generated when the moving average crosses above or below the threshold levels. It calculated as seen in (4).

$\operatorname{MRSI}_{(i)}=\frac{\sum_{i=1}^{n} R S I(i)}{n}$

Where $n$ is the period for calculating the average, RSI is RSI values for day $i$.

\section{PROPOSED MODEL}

\subsection{Encoding of Genetic}

Genetic algorithm is used to optimize parameters for four indicators (RSI, DEMAC, MRSI and MACD)

MACD: The parameters to be optimized for the MACD indicator are and EMA short and EMA long.

RSI: The parameters to be optimized for the RSI indicator are (n) which is the period of used for calculating the RSI, the lower and the upper boundaries.

MARSI: The parameters to be optimized for the MARSI are (P) which is the period used for calculating the RSI, P1 which is the period used for averaging the RSI, the lower and the upper boundaries.

DEMAC: The parameters optimized for the DEMAC are the EMA short and EMA long.

chromosome is composed of four main parts, each part used for one of the indicators. real numbers are used to represent values of different parameters.

Table 1 represent a sample of chromosome values. First part is consisting of 3 gens that represent the MACD indicator (EMA short, EMA long and Signal), second part is consisting of 3 gens that represent RSI indicator (number of periods, lower and upper boundaries), third part is consists of 2 gens that represent DEMC indicator (EMA short and EMA long), finally part is consists of 4 gens that reparents MRSI indicator (first 3 gens are represent same values of RSI gens and 4th gen is represent the period value).

Table 1 optimized indicators chromosome

\begin{tabular}{|l|c|l|l|c|c|c|c|c|c|c|c|}
\hline \multicolumn{3}{|c|}{ MACD } & \multicolumn{3}{c|}{ RSI } & \multicolumn{4}{c|}{ DEMC } & \multicolumn{3}{c|}{ MRSI } \\
\hline 20 & 50 & 12 & 26 & 9 & 14 & 30 & 70 & 26 & 9 & 14 & 14 \\
\hline
\end{tabular}

\section{Genetic algorithm parameters}

Optimization of system is based on 2 of objective functions, sharp ration and annual profit. a genetic algorithm had been evaluated by evaluated vector, it adopted as the multi objective optimization method. the first step, population divided into subpopulations, second step, the selection for each sub-population is based on objective function then recombination for crossover and mutation has been done every 10 iterations, finally the performance is evaluated by summation for weighted sumapproach in order to prevent the solutions from converging entirely towards only one of the objectives.

Table 2 represents the parameters of GA, that used in this experimental.

Table 2 GA Parameters in proposed model

\begin{tabular}{|l|l|}
\hline Population & 1000 \\
\hline No. of iteration & 1000 \\
\hline Selection technique & ranking \\
\hline Probability of crossover & 0.85 \\
\hline Mutation & 0.5 \\
\hline
\end{tabular}

\subsection{Sharpe ratio Function}

Equation (5) presented, The Sharpe proportion was created by Nobel laureate William F. Sharpe and is utilized to assist speculators get it the return of a speculation compared to its risk. The proportion is the normal return earned in overabundance of the risk-free rate per unit of instability or total risk. Instability may be a degree of the cost changes of a resource or portfolio. Subtracting the risk-free rate from the cruel returnpermits a financial specialist to way better confine the benefits related with risk-taking exercises.

The risk-free rate of return is the return on a speculation with zero chance, meaning it's the return financial specialists might anticipate for taking no risk. [17] [18]

$$
\begin{aligned}
& \text { SharpRatio }=\frac{R_{p}-R_{f}}{\delta_{p}}(5) \\
& R_{p} \text { is return of portfolio } \\
& R_{f} \text { is risk free rate } \\
& \delta_{p} \text { is standard deviation of the portfolio return }
\end{aligned}
$$$$
\text { Were }
$$

\subsection{Annual Return}

Annual return is computed by (6), the annualized return of an investment held for a specified number of years.

$$
\begin{aligned}
& \text { Anualreturn }=\left[\left(\frac{\text { Ending value of investment }}{\text { Beginning value of investment }}\right)^{1 / \text { Number days held }}\right]-1(6) \\
& \text { Note: } \\
& \quad \text { Numberdaysheld }=\text { numberoftradingdays } / 260
\end{aligned}
$$

This researchfocusses on long term venture technique. Only within the starting of the venture prepare an initial invested capital is implanted into the advertise. No more cash is implanted amid the speculation period.This implies that each exchange influences all its devotees. In the event that the current exchange is a profitable one, at that point the contributed capital increments (benefits are used for reinvestment within the next exchanges). On the off chance that the current exchange may be a non-beneficial one, at that point the contributed capital diminishes which moreover influences the taking after trades. In expansion, exchanging is administered by the taking after rules: No two progressive buying or offering signals are permitted (each buying flag is taken after by an offering flag). The number of buys and offers are equal. The advantage of this prepare is that it looks at more solidly the capability of the prepared parameters to hold on amid all the speculation periods. 


\subsection{Proposed model algorithm}

Following algorithm represent an algorithm

Strat

Initiate random publication

While number of iterations

begin

Do while iteration less than $10 \%$ of population size

begin sharp ratio

Divide population into 2 sub-population

Compute and evaluate sub-population based on

Select second sub-population according evaluating using sharp

Compute and evaluate second sub-population based annual profit

Combination for sub-population into one population

Evaluate the population against the two fitness functions

Select the population according to the weighted sum of both

fitness functions

end

Crossover and Mutation

end

\section{PROPOSED MODEL RESULTS}

The proposed model is related to the closing price of EURUSD. The optimized parameters are evolved on the information (From $1 / 1 / 1990$ to $1 / 1 / 2020) 7800$ exchanging days. When optimizing the parameters, GA tends to form the highest returns within the preparing period. In some cases, GA as all other optimization strategies may endure from over fitting and the gotten parameters may not give any benefits in other periods. So that, the part of arrangement into preparing, validation, and testing periods in arrange to discover vigorous arrangements is presented.Also the rolling forward strategy in which the information series is part into 7 groupings is used.

Each grouping is spoken to as an independent run.In each grouping the first 10 a long time are takenas preparing period, the another three a long time are taken as the approval period and the last three a long time are the testing period. The information in one sequence overlapsthe information withinthe following arrangement.

For each sequence we move all the periods three a longtime forward.

Thedata arrangement is separated into seven groups called (A,B,C,D,E,F,andG).

The moment half of the preparing period with the entire approval periodconstitute the another preparing period. The testing period in one arrangement ended up the validation period within the following grouping and so on.

For case arrangement. Group 'A', The training phase is starting from $1 / 1 / 1990$ to $31 / 12 / 2000$, follows by validation phase is starting from $1 / 1 / 2001$ to $31 / 12 / 2005$ finally testing phase is starting from $1 / 1 / 2006$ to $31 / 12 / 2010$, Sequentially other groups. The valuation is separated into 3 of phases (training, validation, and testing). For each iteration the parameters are firstly optimized over the training phase. The ultimate populace is reused as a beginning populace for the validation phase. At that point the ultimate populace of the evaluation phase is reevaluated against the training phase and organized concurring to the in general average gain of both preparing and evaluation phase. The finest obtained parameters that coordinate both the preparing and evaluation phase are at that point at long last tried within the testing phase.
In this research, researcher apply model in two of trading strategies (random strategy and buy or sell-hold strategy).

\subsection{Random strategy}

The comparing in random strategy, had been executed by 50 independent iterations. For each iteration the annual return and the Sharpe ratio is calculated, then obtained results are averaged for all training, validation and testing phases.

Fig. 4and Fig. 5are represent, competing for average of annual return and random strategy, average of sharp ratio and random strategy in sequential.

Performance evaluation is tested by comparing results for buysell and hold. Wherein the forex market you can exchange from the Euro to Dollar.

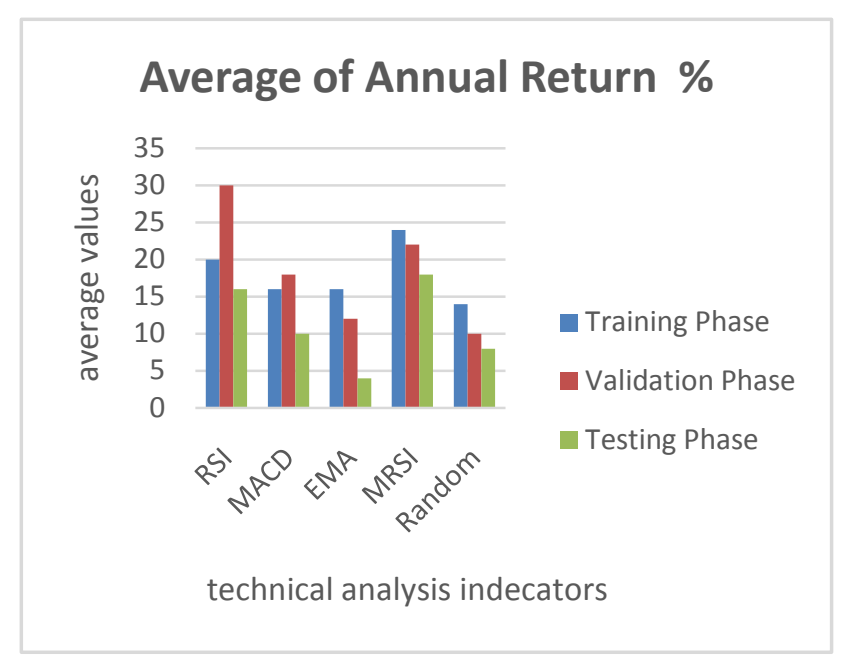

Fig. 4 Compering Average annual return for the optimized indicators and the random strategy

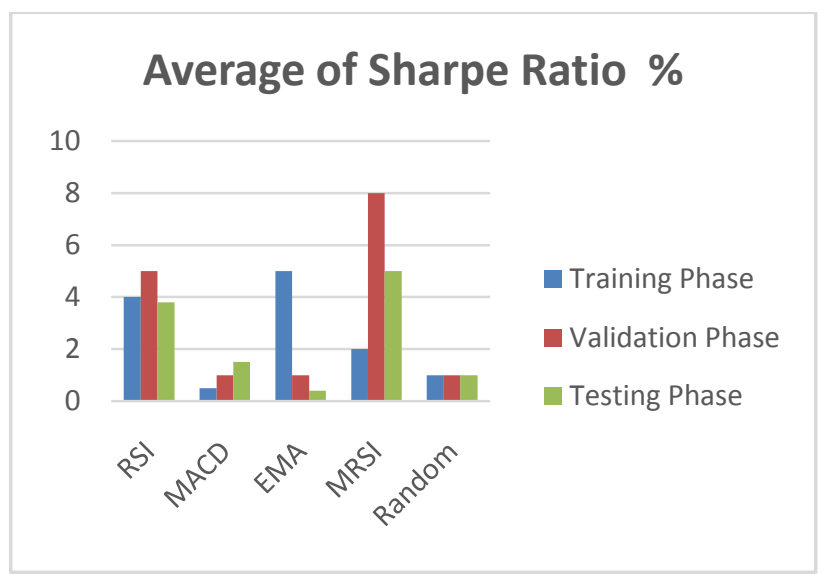

Fig. 5 Comparing Average Sharpe ratio for the Optimized Indicators vs. the Random Strategy

\subsection{Buy or Sell-hold Strategy}

The strategy is built on join the market by exchange (buy or sell) then hold till the end of the period, also indicators (RSI, MACD, MRSI, and EMA) are taken, finally a comparison between results and random strategy is executed. the implementation has been executed 50 iterations. each iteration is run for groups that had been presented. Table 3 and table 4 are represent the results for the nearest optimum and the average for training, evaluation, and testing phases for all indicators. 
Table 3 The average performance of the RSI indicator

\begin{tabular}{|l|l|c|c|c|}
\hline \multicolumn{2}{|c|}{} & Best & Average & $\begin{array}{c}\text { Sell, Buy and } \\
\text { hold }\end{array}$ \\
\hline \multirow{2}{*}{$\begin{array}{l}\text { Training } \\
\text { Phase }\end{array}$} & Return (\%) & 9.829 & $\mathbf{8 . 3 6 6}$ & 11.176 \\
\cline { 2 - 5 } & Sharpe & $\mathbf{0 . 2 0 5}$ & $\mathbf{0 . 1 9 0 8 2}$ & ---- \\
\hline \multirow{2}{*}{$\begin{array}{l}\text { Evaluation } \\
\text { Phase }\end{array}$} & Return (\%) & $\mathbf{9 . 7 9 5}$ & $\mathbf{9 . 6 7 4 1 5}$ & 7.627 \\
\cline { 2 - 5 } & Sharpe & $\mathbf{0 . 2 0 5}$ & $\mathbf{0 . 3 2 2 4 1}$ & ----- \\
\hline \multirow{2}{*}{$\begin{array}{l}\text { Testing } \\
\text { Phase }\end{array}$} & Return (\%) & $\mathbf{7 . 4 1 4}$ & $\mathbf{5 . 3 5 1 5 8}$ & 7.800 \\
\cline { 2 - 5 } & Sharpe & $\mathbf{0 . 1 8 1}$ & $\mathbf{0 . 1 7 3 2 3}$ & ----- \\
\hline
\end{tabular}

Table 4 The average performance of the DEMAC

\begin{tabular}{|l|l|c|c|c|}
\hline \multicolumn{2}{|c|}{} & Best & Average & $\begin{array}{c}\text { Sell, Buy } \\
\text { and hold }\end{array}$ \\
\hline \multirow{2}{*}{$\begin{array}{l}\text { Training } \\
\text { Phase }\end{array}$} & Return (\%) & 8.038 & 8.141 & 11.176 \\
\cline { 2 - 5 } & Sharpe & 2.997 & 2.446 & ------ \\
\hline \multirow{2}{*}{$\begin{array}{l}\text { Evaluation } \\
\text { Phase }\end{array}$} & Return (\%) & 7.286 & 6.399 & 7.6275 \\
\cline { 2 - 5 } & Sharpe & 0.535 & 0.211 & ----- \\
\hline \multirow{2}{*}{$\begin{array}{l}\text { Testing } \\
\text { Phase }\end{array}$} & Return (\%) & 4.895 & 2.153 & 7.8006 \\
\cline { 2 - 5 } & Sharpe & 0.547 & 0.0461 & ------ \\
\hline
\end{tabular}

\section{CONCLUSION}

The parameters of Stock market indicators are optimized using Genetic Algorithm. Maximize profit is a target for annual return and sharp ratio, is studied in this research. Proposed model is applied for EUR/USD in forex market. Data is collected from "Metatrader 4" application for forex market. Data is separated into training, evaluation and testing phases. In buy or sell and hold strategy, 3 inductors (RSI, MARSI and MACD) results are best in buy or sell and hold strategy.

DEMAC was the as it were marker that seem not beat the advertise that's since the nature of the moving normal pointers as slacking markers. Comparisons between optimized indictors and random strategy accepted that optimized indicators are better.

An optimum chromosome for technical indicator parameter is used as input to fuzzy interference system, to predive stock mark. Predictive system is based on fuzzy logic.

\section{REFERENCES}

[1] S. Taylor., Modeling Financial Time Series, John Wiley \& Sons , 1986.

[2] B. G. Malkiel, A Random Walk Down Wall street 6th Ed., W.W. Norton \& Company, 1973.

[3] J. J. Murphy, TECHNICAL ANALYSIS OF THE GUIDE TO TRADING METHODS AND APPLICATIONS, New York Institute of Finance, 2009.

[4] A. W. Teeples, "An Evolutionary Approach to
Optimization of Compound Stock Trading Indicators Used to Confirm Buy Signals," p. Paper 820, 2010.

[5] M. D. a. C. M. Jones., "The profitability of intra-day FX trading using technical indicators," University of Cambridge Judge Institute of Management Studies, 2010.

[6] C. A. a. H. I. Akinori Hirabayashi, "Optimization of the Trading Rule in Foreign Exchange using Genetic Algorithm," GECCO, 2009.

[7] S.-k. L. a. B.-R. Moon, "Finding attractive rules in stock markets using a modular genetic programming," GECCO, 2009.

[8] D. F. a. J. P.Fernandez, "Technical market indicators optimization using EA," GECCO, 2008.

[9] R. F. N. N. H. Adriano Simões, "An Innovative GA Optimized Investment Strategy based on a New Technical Indicator using Multiple MAS," in International Joint Conference on Computational Intelligence IJCCI 2010, Valencia,Spain, 2010.

[10] S. a. A. K. V.Kapoor, "Genetic Algorithm: An Application to Technical Trading System Design," International Journal of Computer Applications (0975 - 8887), vol. 36- No.5, 2011.

[11] V. C. L. a. Y. S. O. Devayan Mallick, "An Empirical Study of Genetic Programming Generated Trading Rules in Computerized Stock Trading Service System," IEEE proceedings, 2018.

[12] M. Becket, How the stock market works, London: Kogan Page Limited, 2014.

[13] M. Heitkoetter, The complete guide to day trading, USA: BookSurge, LLC, 2014.

[14] M. J. Pring, Technical Analysis for Short-Term Traders, Traders' Library, 2000.

[15] S. a. A. K. V.Kapoor, "Genetic Algorithm: An Application to Technical Trading System Design," International Journal of Computer Applications, vol. 36, no. 5, 2011.

[16] C. M. Brown, Tecincal Analysis for Trading Profissional, USA: The McGraw Hill, 2012.

[17] M. C. Weigend, "Nonlinear Trading Models Through Sharpe Ratio Maximization," Leonard N. Stern School of Business, 2012.

[18] A. S. Weigend, "Decision Technologies for Financial Engineering," in Fourth International Conference on Neural Networks in the Capital Markets,NNCM, 2015.

[19] E. J. M. \&. S. M. Alejandro, "A Technical Analysis Indicator Based on Fuzzy Logic," Electronic Notes on Theoretical Computer Science, vol. 292, pp. 27-37, 2013.

[20] P. S. Hussein Dourraa, "Investment using Technical Analysis and Fuzzy Logic. Fuzzy Sets and Systems," Fuzzy Sets and Systems, vol. 127, no. 2, pp. 221-240, 2012.

[21] J. F. Yates, Judgment and Decision Making, Prentice-Hall, Englewood Cliffs, NJ, 1990 